\title{
Persistent Complete Heart Block in a Patient with COVID-19 Infection: a Case Report
}

\author{
Zahra Hosseini $^{1} \cdot$ Saeed Ghodsi ${ }^{1,2}$ (I) $\cdot$ Seyed Fakhreddin Hejazi ${ }^{1}$
}

Accepted: 10 December 2020 / Published online: 6 January 2021

(C) Springer Nature Switzerland AG 2021

\begin{abstract}
The pandemic of COVID-19 as a global concern has emerged the need for data aggregation about various clinical pictures particularly cardiovascular complications. Although the incidence of advanced atrioventricular block (AVB) in these patients is not well established, few cases have been reported. We have reported a 48-year-old man with COVID-19 infection who presented with prodromal symptoms for 5 days preceding complete AVB found at the emergency department. Pulmonary involvement and PCR confirmed the diagnosis. The block persisted after recovery of the patient for more than 1 month. Pathophysiology of advanced AVB following COVID-19 infection is not well understood. Several factors including inflammatory response, immune system over activity, myocarditis, and medications have been underlined. Although conservative management may lead to spontaneous recovery of $\mathrm{AVB}$, pacemaker implantation is reasonable in case of persistent conduction defect.
\end{abstract}

Keywords COVID-19 $\cdot$ SARS-CoV-2 $\cdot$ Infection $\cdot$ Atrioventricular block $\cdot$ Inflammation $\cdot$ Pacemaker

\section{Introduction}

Coronavirus outbreak started in late 2019 in Wuhan, China. Over a short time, it turned to a striking pandemic [1]. Unfortunately, this is a new viral disease, and we do not know much about it. Although respiratory tract involvement including pneumonia is found as the most common manifestation, cardiac features are also emerging complications. A study in Wuhan reported the frequency of acute myocardial damage about $7.2 \%$ and cardiac arrhythmias about $16.7 \%$. However, severe bradyarrhythmias such as third-degree atrioventricular block (AVB) have been relatively uncommon [2]. Herein, we

This article is part of the Topical Collection on Covid-19

Saeed Ghodsi

dsaeedgh@gmail.com

Zahra Hosseini

Zmed702@yahoo.com

Seyed Fakhreddin Hejazi

sf-hejazi@ muq.ac.ir

1 Department of Cardiology, Shahid Beheshti Hospital, Qom University of Medical Sciences, Qom, Iran

2 Research Department, Tehran Heart Center, Tehran University of Medical Sciences, North Kargar Street, Tehran 14111713138, Iran have described the clinical presentation of advanced AVB in a patient with uncomplicated COVID-19 infection, which persisted over 4 weeks without hemodynamic instability.

\section{Case Presentation}

The patient was a 48-year-old man who was previously healthy with a negative past medical history. He came to our emergency department with chief complaint of fever and chills. He also mentioned weakness, lethargy, myalgia, and cough since about 4 or 5 days ago. His initial vital signs were recorded as follows: low-grade fever (T: $\left.37.6^{\circ} \mathrm{C}\right)$; blood pressure, $124 / 67 \mathrm{mmHg}$; heart rate, 42 beats per minute (bpm); and a respiratory rate of $20 \mathrm{bpm}$. Initial oxygen saturation was detected which showed a low level $(88 \%)$ indicating to hypoxemia.

Due to the COVID-19 epidemic, we performed laboratory tests including CBC, C-reactive protein (CRP), as well as spiral chest $\mathrm{CT}$ scan. Imaging revealed a severe parenchymal involvement of the lungs (Fig. 1a and b). Diffuse patchy infiltrations and ground glass appearance were evident. Laboratory tests showed a markedly increased CRP level, leucocytosis, and relative lymphocytopenia. White blood cell count (WBC), lymphocyte count, platelet count, and haemoglobin level were $10,200 / \mu \mathrm{L}, 1150 / \mu \mathrm{L}, 190,000 / \mu \mathrm{L}$, 

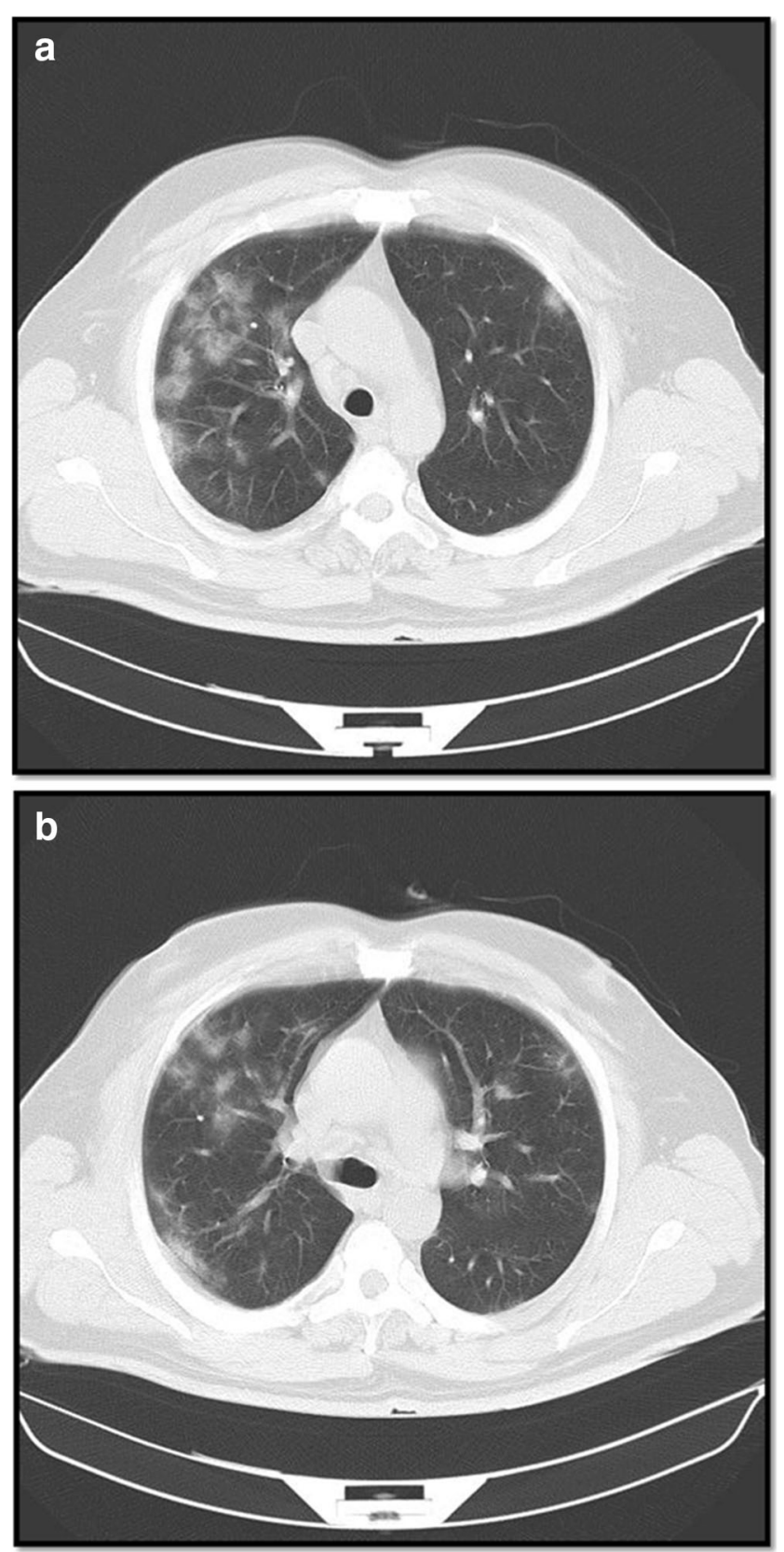

Fig. 1 This montage illustrates the widespread involvement of both lungs. Ground-glass appearances in concert with diffuse patchy infiltrations predominantly seen in the peripheral (and sub-pleural) parts are evident

and $12.8 \mathrm{~g} / \mathrm{dl}$, respectively. CRP level and erythrocyte sedimentation rates after $1 \mathrm{~h}($ ESR $1 \mathrm{~h})$ were $11.9 \mathrm{mg} / \mathrm{L}$ and $48 \mathrm{~mm} / \mathrm{h}$, respectively. CRP level increased up to 14.3 over 5 days and then reached a steady state until a fall was detected since day $10(7.3 \mathrm{mg} / \mathrm{L})$.

Based on the patient's history and clinical findings, we started treatment with azithromycin and hydroxychloroquine. During regular daily ECG monitoring, QT intervals were measured which did not exceed the upper limit of normal values. A nasopharyngeal swab specimen was obtained in order to analyse with PCR. The PCR assay confirmed the diagnosis of COVID-19 infection. Given the patient's low heart rate of approximately 42, an ECG was taken which demonstrated advanced atrioventricular block (Fig. 2a and b). Our patient had no history of prior known medical disorders as well as taking any medications possibly affecting cardiac conduction. Renal and liver function tests were normal $(\mathrm{Cr}=1 \mathrm{mg} / \mathrm{dL})$. Electrolytes were also found within normal ranges $(\mathrm{K}=$ $4.1 \mathrm{mmol} / \mathrm{L}, \mathrm{Mg}=2.5 \mathrm{mmol} / \mathrm{L}, \mathrm{Ca}=8.6 \mathrm{mg} / \mathrm{dL}$ ). The patient underwent trans-thoracic echocardiography. Left ventricular ejection fraction was normal (55\%) without significant valvular heart disease or pericardial effusion. We found nothing in favour of infective endocarditis including vegetations and perivalvular abscess. Biomarkers of myocardial injury such as troponin and CKMB were negative. Measured troponin-I concentration and CKMB levels were $<1.5 \mathrm{ng} / \mathrm{L}$ and $14 \mathrm{IU} / \mathrm{L}$, respectively.

After 7 days, the patient showed signs of improved hemodynamic, and nutrition. Pulse oximetry revealed a promising trend (90-93\%). After 10 days, inflammatory markers also tended to decline, and the patient appeared better. According to electrophysiology consultation, considering that the patient's hemodynamic was stable and free of CHB-related symptoms, implantation of the permanent pacemaker (PPM) was postponed. A reasonable expectation was spontaneous recovery of the AV node function following the decline of inflammation. However, complete heart block persisted after 4 weeks, and the patient became a candidate for PPM implantation.

\section{Discussion}

Since we have limited knowledge regarding pathophysiology of cardiovascular complications related to COVID-19, a clear picture of conduction defects is still missing. There are different hypotheses suggested to explain the mechanism of AVB in this setting. First, an immense myocardial damage either due direct viral infiltration or secondary to hypoxemia is thought to cause conduction defect [3, 4]. Moreover a previous study revealed that SARS-CoV-2 virus RNA was discovered in the myocardial tissue of $35 \%$ of infected individuals [5]. However, overt myocardial injury defined as a significant elevation of highly sensitive troponin was not confirmed in this patient. Second, the presence of severe acute respiratory distress syndrome (SARS) might entail new advanced blocks, which also afford poor clinical outcome. Furthermore, SARS was observed in majority of the cases presented with advanced AVB highlighting the prognostic implication of this finding $[6,7]$. Nonetheless, our patient did not show features compatible with the aforementioned severe type of the disease. Third, increased expression of the angiotensin-converting enzyme 2 receptor (ACE-2) due to SARS-CoV-2 may also 


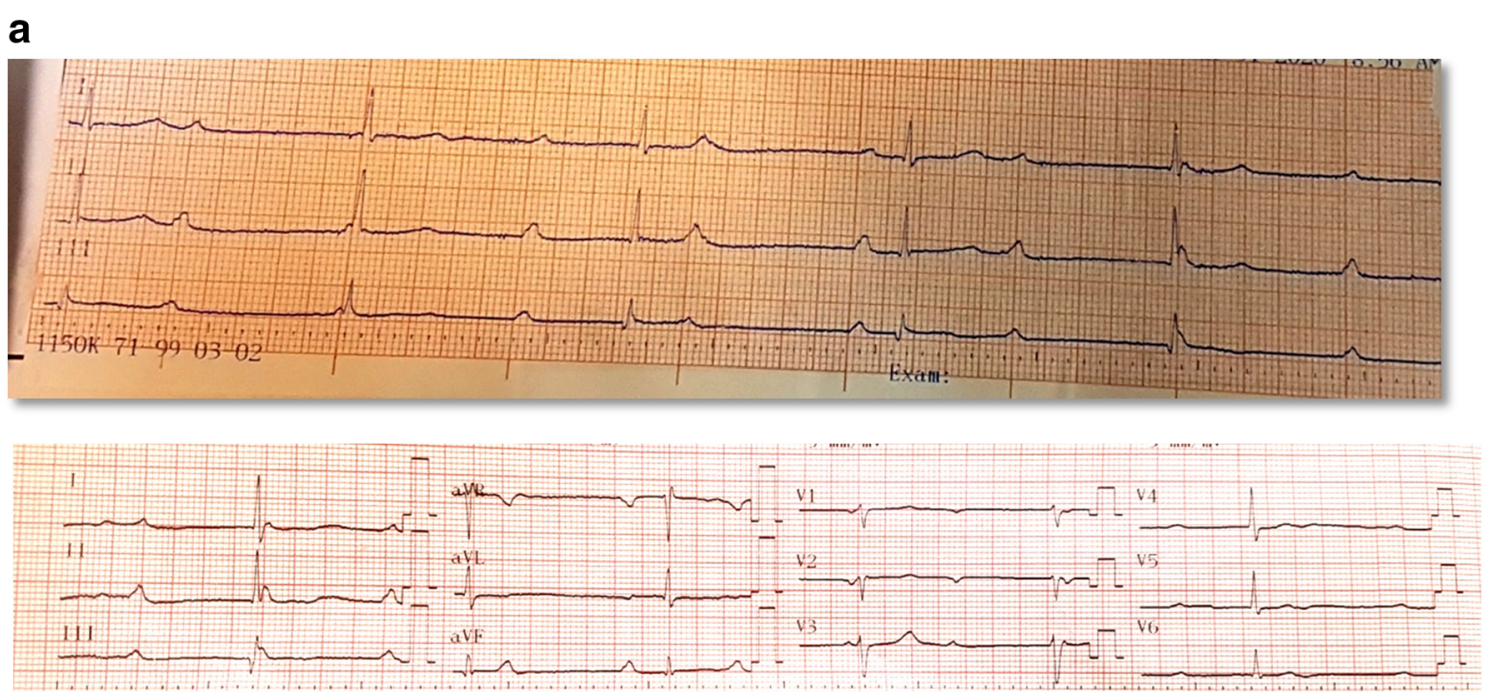

b

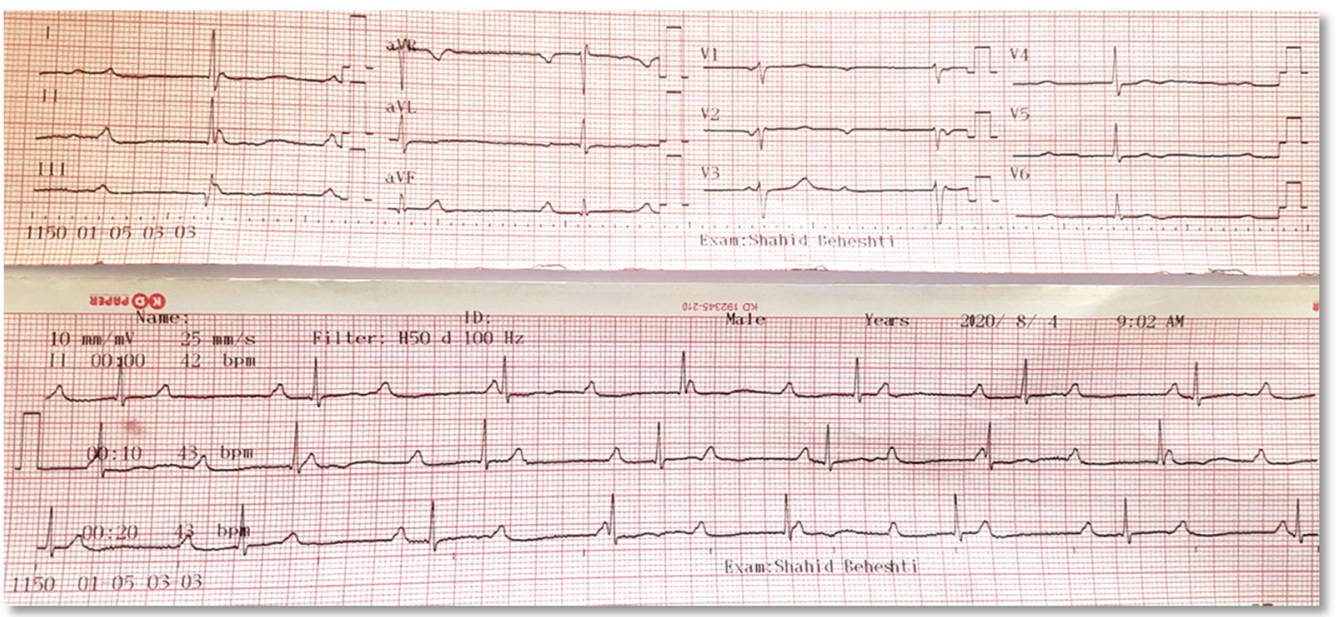

Fig. 2 a First ECG tracings show complete atrioventricular block at initial visit. Furthermore, normal QRS axis, escape rhythm with narrow QRS complexes, and no significant ST-T changes are observed. b

compromise normal conduction. However, this finding mainly contributes to sinus node dysfunction rather than AVB [8]. Likewise, a remarkable activation of immune response including cytokine/chemokine storm as well as amplification of the inflammatory cascade occurs [9]. Exacerbation of the preexisting conduction disorders such as bundle branch blocks, AV node disease, or His-Purkinje fibre's disease is likely to cause advanced blocks. Moreover, infrequent reports have raised caution regarding aggravation of AVB following initial doses of azithromycin and hydroxychloroquine [10] as well as their long-term use [11]. This hypothesis also seems unlikely in our patient because the advanced AVB was observed prior to receiving these medications.

In brief, we encountered an advanced AVB as the major involvement of a COVID-19 infection beside pneumonia. Although we thought that the systemic inflammation in conjunction with hypoxemia and a transient hypercoagulable state
Electrocardiographic pattern is relatively unchanged after 4 weeks indicating to persistent advanced AV block

might explain the incident AVB, the puzzle has still missing parts. First, myocardial involvement was not confirmed. Maybe a subclinical or focal injury has occurred in this case. Second, following the recovery of oxygenation and reduced inflammation, the block was persistent. Since the QRS, complexes are narrow, and the rate of the escape rhythm approximates to or exceeds $40 \mathrm{bpm}$; infranodal damage seems unlikely. In the same way, diffuse injury of the distal small conducting fibres also appears implausible. Furthermore, the latter requires an evident full blown myocarditis. We found only one report with similar presentation of advanced AVB in non-severe stable COVID-19 patients [6]. However, the outstanding feature of our patient was persistence of third-degree AVB despite recovery of the COVID-19 infection as well as decreasing inflammation. This might address direct viral injury or prolonged antibody-mediated insult. A plausible hypothesis may be selective involvement of the AV node or the 
adjacent tissues by the virus or inflammatory factors in order to cause damage or electrical remodelling. Although the complications of a viral infection may last for a long time, solid data lacks regarding prolonged $\mathrm{AVB}$ as well as the appropriate management.

Acknowledgments The authors appreciate the companionship of the nurses in emergency ward and CCU of Shahid Beheshti Hospital.

Authors' Contributions ZH and SFH presented the core concept of the case report and performed treatment of the patient as well as supervising the study. ZH collected the data. ZH and SG wrote the draft and revised the manuscript. $\mathrm{ZH}$ and SG, prepared the figures. All authors read and approved the final manuscript.

Data Availability Not applicable to this type of study (case report). Data sharing not applicable to this article as no datasets were generated or analysed during the current study.

\section{Compliance with Ethical Standards}

Competing Interests The authors declare that they had no competing interests.

Ethics Approval The patient consented for publication of the case report. It was not needed for case reports at Tehran Heart Center. Case reports are exempted from the ethical approval in Tehran Heart Center. However, codes of the ethics guideline of Tehran University of Medical Sciences were met in this study.

Informed Consent We obtained written informed consent from the patient for publishing the clinical data and related anonymous images. A copy of the written consent is available for review in order to present in case of request by editorial board of the journal.

Consent for Publication Not applicable.

Code Availability Not applicable.

\section{References}

1. World Health Organization. Coronavirus disease (COVID-19) situation reports.https://www.who.int/emergencies/diseases/novelcoronavirus-2019/situation-reports/.Google Scholar.

2. Chinitz JS, Goyal R, Harding M, Veseli G, Gruberg L, Jadonath R, et al. Bradyarrhythmias in patients with COVID-19: marker of poor prognosis? Pacing Clin Electrophysiol. 2020;43(10):1199-204. https://doi.org/10.1111/pace.14042.

3. Bansal M. Cardiovascular disease and COVID-19. Diabetes Metab Syndr. 2020;14(3):247-50. https://doi.org/10.1016/j.dsx.2020.03. 013.

4. Pishgahi M, Yousefifard M, Safari S, Ghorbanpouryami F. Electrocardiographic findings of COVID-19 patients and their correlation with outcome; a prospective cohort study. Adv J Emerg Med. https://doi.org/10.22114/ajem.v0i0.470.

5. Oudit GY, Kassir Z, Jiang C, et al. SARS-coronavirus modulation of myocardial ACE2 expression and inflammation in patients with
SARS. Eur J Clin Investig. 2009;39(7):618-25. https://doi.org/10. $1111 / \mathrm{j} .1365-2362.2009 .02153$.

6. Babapoor-Farrokhran S, Rasekhi RT, Gill D, Babapoor S, Amanullah A. Arrhythmia in COVID-19. SN Compr Clin Med. 2020:1-6. https://doi.org/10.1007/s42399-020-00454-2.

7. Mahdawi TE, Wang H, Haddadin FI, Al-Qaysi D, Wylie JV. Heart block in patients with coronavirus disease 2019: a case series of 3 patients infected with SARS-CoV-2. Heart Rhythm Case Rep. 2020;6(9):652-6.

8. Kochi A, Tagliari AP, Forelo B, Fassini GM, Tondo C. Cardiac and arrhythmic complications in patients with COVID-19. J Cardiovasc Electrophysiol. 2020;21:1003-8.

9. Lazzerini PE, Boutjdir M, Capecchi PL. COVID-19, arrhythmic risk and inflammation: mind the gap! Circulation. 2020. https:// doi.org/10.1161/CIRCULATIONAHA.120.047293.

10. Javorac D, Grahovac L, Manić L, Stojilković N, Anđelković M, Bulat Z, et al. An overview of the safety assessment of medicines currently used in the COVID-19 disease treatment. Food Chem Toxicol. 2020;144:111639. https://doi.org/10.1016/j.fct.2020. 111639.

11. Chatre C, Roubille F, Vernhet H, Jorgensen C, Pers YM. Cardiac complications attributed to chloroquine and hydroxychloroquine: a systematic review of the literature. Drug Saf. 2018;41(10):919-31. https://doi.org/10.1007/s40264-018-0689-4.

\section{Learning Points:}

1. Bradyarrhythmias including advanced AV block are uncommon in COVID-19 infection but indicate to complicated course of the disease and poor prognosis. Complete AVB might rarely occur in patients with nonsevere stable patients, which should be acknowledged.

2. In case of incident advanced AVB a wide variety of aetiologies might be addressed, however, inflammation is thought to be a major culprit. Although persistent AVB after treatment of underlying stable COVID-19 infection is rare, PPM implantation is an appropriate choice.

Timeline:

\begin{tabular}{ll}
\hline Time & Events \\
Day 0 & Prodromal symptoms \\
Day 5 & Index admission \\
Day 5 (at initial visit) & Documented diagnosis AV block \\
$\begin{array}{l}\text { Day 5 (4 h after } \\
\text { admission) }\end{array}$ & Documented pneumonia in chest CT scan \\
Day 5 (8 h after & Initial treatment with azithromycin and \\
admission) & hydroxychloroquine \\
Day 6 & Confirmed diagnosis of COVID-19 via PCR \\
Day 18 & Termination of dedicated treatment course \\
Day 21 & Discharge \\
Day 33 & Last outpatient follow-up visit (persistent AVB) \\
Day 41 & PPM implantation \\
\hline
\end{tabular}

Publisher's Note Springer Nature remains neutral with regard to jurisdictional claims in published maps and institutional affiliations. 